DEPÓSITO LEGAL ZU2020000153

Esta publicación científica en formato digital

es continuidad de la revista impresa

ISSN 0041-8811

E-ISSN 2665-0428

Revista

de la

Universidad

del Tunlia

Fundada en 1947

por el Dr. Jesús Emrique Lossada

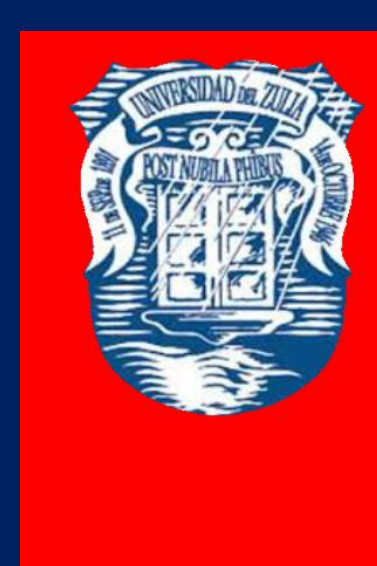

Ciencias

Sociales

y Arte

Aกัต 11 No 31

Septiembre - Diciembre 2021

Tercera ípoca

Maracailbo-Venezuela 
REVISTA DE LA UNIVERSIDAD DEL ZULIA. 3a época. Año $11 \mathrm{~N}^{\circ}$ 31, 2020

Clarita Melissa Puitiza Lucano et al/// Clima organizacional y desempeño laboral ... 71-85

DOI: http://dx.doi.org/10.46925//rdluz.31.06

\title{
Clima organizacional y desempeño laboral en trabajadores de la microred de salud Bagua Grande en Perú
}

\author{
Clarita Melissa Puitiza Lucano* \\ Sonia Tejada Muñoz** \\ Eli Morales Rojas*** \\ Segundo Chávez Quintana ${ }^{* * * *}$ \\ Tito Sánchez Santillan*****
}

RESUMEN

El objetivo de la presente investigación fue determinar la relación entre el clima organizacional y el desempeño laboral en los trabajadores de la Microred de Salud Bagua Grande en Perú, año 2019. Mediante la aplicación de encuestas e instrumentos del Clima Organizacional y el Formato de Evaluación de Desempeño del Ministerio de Salud del Perú (MINSA). El análisis estadístico se realizó mediante la prueba Chi-cuadrado y el coeficiente de correlación de Pearson (Rho). Los resultados indican que el clima organizacional y el desempeño laboral fueron regulares (46.7\% y $71.7 \%$, respectivamente). El clima organizacional se correlacionó significativa y directamente con una intensidad moderada ( $\mathrm{p}=0.001 ; \mathrm{r}$ : 0.417). Las dimensiones del clima relacionado con el desempeño, fue la cultura en la organización ( $\mathrm{p}=0.001 ;$ r: 0.432) y el diseño organizacional ( $\mathrm{p}=0.001$; r: 0.425). Sin embargo, el potencial humano fue la única dimensión no relacionada con el desempeño en las labores ( $\mathrm{p}<0.05$; $\mathrm{r}$ : $0.244)$.

PALABRAS CLAVE: usuario interno, clima organizacional, desempeño laboral, dimensión.

* Egresada de la escuela de posgrado en Gerencia y Gestión de Servicios para la Salud, Universidad Nacional Toribio Rodríguez de Mendoza de Amazonas, Perú. ORCID: https:/orcid.org/0000-00028836-4807. E-mail: cmpuitiza@gmail.com

** Docente asociado a tiempo completo al departamento académico de Salud Pública, Universidad Nacional Toribio Rodríguez de Mendoza de Amazonas. ORCID: https://orcid.org/0000-0002-1181-8540. E-mail: sonia.tejada@untrm.edu.pe

***Investigador del Instituto de Investigación para el Desarrollo sustentable de Ceja de Selva INDESCES, Universidad Nacional Toribio Rodríguez de Mendoza de Amazonas, Perú. ORCID: https://orcid.org/0000-0002-8623-3192. E-mail: eli.morales@untrm.edu.pe

****Investigador del Instituto de Investigación para el Desarrollo sustentable de Ceja de Selva INDESCES, Universidad Nacional Toribio Rodríguez de Mendoza de Amazonas, Perú. ORCID: https://orcid.org/0000-0002-0946-3445. E-mail: segundo.quintana@untrm.edu.pe

***** Investigador del Instituto de Investigaciones de la Amazonía Peruana, Av. Abelardo Quiñones Km 2.5 Iquitos-Loreto, Perú. ORCID: https://orcid.org/0000-0002-3352-341X E-mail: titosanchezsantillan@gmail.com

Recibido: 10/07/2020

Aceptado: 11/09/2020 


\section{Organizational climate and work performance in workers of the Bagua Grande health microgrid in Peru}

ABSTRACT

The objective of this research was to determine the relationship between the organizational climate and work performance in the workers of the Bagua Grande Health Micro-network in Peru, year 2019. Through the application of surveys and instruments of the Organizational Climate and the Evaluation Format of Performance of the Ministry of Health of Peru (MINSA). Statistical analysis was performed using the Chi-square test and Pearson's correlation coefficient (Rho). The results indicate that the organizational climate and job performance were regular (46.7\% and $71.7 \%$, respectively). The organizational climate was significantly and directly correlated with a moderate intensity ( $\mathrm{p}=0.001 ; \mathrm{r}$ : 0.417). The dimensions of the climate related to performance were the culture in the organization $(\mathrm{p}=0.001 ; \mathrm{r}: 0.432)$ and the organizational design $(\mathrm{p}=0.001 ; \mathrm{r}$ : 0.425$)$. However, human potential was the only dimension not related to job performance ( $\mathrm{p}<0.05 ; \mathrm{r}: 0.244)$.

KEYWORDS: internal user, organizational climate, job performance, dimension.

Introducción

La Organización Mundial de la Salud ha reconocido el desempeño laboral en salud en la manera en que un colaborador realiza sus tareas, nivel de cumplimiento de las exigencias del puesto laboral en concordancia con los objetivos organizacionales, siendo un factor que impacta inmediatamente sobre la asistencia de servicios sanitarios, así como en la población (Gonzáles,2006). Múltiples investigaciones reconocen que existen muchos factores que influyen en el desempeño de los trabajadores de salud, desde factores institucionales tales como la dotación de recursos materiales hasta factores individuales del trabajador sanitario (Sharma et al, 2014; Weldegebriel et al, 2016; Irimu et al, 2014). Los factores psicosociales como el clima del entorno laboral parecen influir consistentemente sobre el desempeño laboral sanitaria (Tran et al, 2018). Por ende, recomiendan evaluar la relevancia y viabilidad de estrategias para determinar el vínculo entre clima y desempeño laboral antes de su implementación (Ballard \& Montgomery, 2017). 
Los niveles óptimos del desempeño laboral son una necesidad en el marco de las prestaciones sanitarias; sin embargo, no existen reportes de su cuantificación y menos aún si el clima laboral es una de sus causales. Ello es trascendental por cuanto la determinación del desempeño laboral actual mediante metodologías del Ministerio de Salud del Perú (MINSA) y la evaluación de su relación con el clima laboral permitirán una mejor planificación de intervenciones futuras, volviéndose importante para el sector salud, tanto privadas como nacionales, asegurando su sostenibilidad en el tiempo. Peña et al. (2015), menciona también que el estilo directivo no influye sobre el desempeño organizacional. En otros estudios han reportado situaciones no muy favorables en cuanto a clima laboral investigaciones a nivel hospitalario encuentran situaciones medianamente desfavorables, especialmente en lo referido a diseño organizacional y potencial humano (Solís, 2017; Picoy, 2015; Espinoza, 2018; García, 2016).

Para Aliaga \& Julcamoro (2016) el clima institucional de una organización si influye sobre el desempeño del personal del área de hospitalización de un establecimiento público, teniendo que mejorar el clima organizacional en un hospital tomando en cuenta la opinión de los trabajadores. La Microred de Salud Bagua Grande forma parte de la Red de salud Utcubamba y la conforman 13 establecimientos de salud, los cuales brindan prestaciones sanitarias a la población adscrita a su jurisdicción. En documentos oficiales se han reconocido insuficientes mecanismos de monitoreo y evaluación del desempeño; de ahí que instrumentos de gestión indiquen la orientación a la promoción del crecimiento del desempeño organzativo-institucional de acuerdo a las metas programadas (Gobierno Regional de Amazonas, 2015). Así, lograr óptimos niveles de desempeño laboral son una necesidad en el marco de las prestaciones sanitarias; sin embargo, no existen reportes de su cuantificación. Es importante mencionar que en la microred de Bagua Grande es la primera vez que se realiza un estudio como este; asimismo, pocas veces se investiga la relación entre el clima organizativo y el desempeño laboral en entornos macroasistenciales.

Por tanto, el objetivo de investigación fue determinar la relación entre el clima organizacional y el desempeño laboral en los trabajadores de la Microred de Salud Bagua Grande, 2019. 


\section{Materiales y Métodos}

Esta investigación tuvo un diseño correlacional, dado que buscó medir la relación de los atributos del clima organizacional y el desempeño laboral. El estudio es de tipo no experimental, al no manipularse las variables basándose en la observación de los fenómenos de manera natural para su análisis. La población general estuvo compuesta por sesenta y ocho (68) trabajadores (administrativos y asistenciales) que actualmente laboran en la Microred de Salud Bagua, Perú (Tabla 01).

Tabla 01: Constitución de la población para la presente investigación

\begin{tabular}{|c|c|c|c|c|}
\hline Tipo de trabajador & Total & $\begin{array}{c}\text { Tiempo laboral } \\
\text { 3 meses }\end{array}$ & $\begin{array}{c}\text { En situación } \\
\text { de licencia }\end{array}$ & $\begin{array}{c}\text { En situación } \\
\text { de } \\
\text { vacaciones }\end{array}$ \\
\hline Médico & 07 & 0 & 0 & 0 \\
\hline Enfermera & 12 & 1 & 0 & 1 \\
\hline Obstetra & 09 & 0 & 0 & 1 \\
\hline $\begin{array}{c}\text { Cirujano Dentista } \\
\text { Administrativo } \\
\text { (tec. Computación } \\
\text { etc.) }\end{array}$ & 02 & 0 & 0 & 0 \\
\hline $\begin{array}{c}\text { Técnico } \\
\text { (enfermería, } \\
\text { laboratorio) }\end{array}$ & 33 & 0 & 0 & 02 \\
\hline Vigilante & 01 & 0 & 0 & 0 \\
\hline Chofer & 01 & 0 & 0 & \\
\hline Total & 68 & 1 & 0 & 4 \\
\hline
\end{tabular}

Muestra: Estuvo constituida por 60 profesionales de salud que laboraban en la Microred de Salud Bagua Grande en el periodo de diciembre de 2019. Asimismo, en la toma de la muestra se consideró los siguientes criterios de selección: a) Profesionales sanitarios (médicos y no 
médicos) y auxiliares; b) Aquellos que contaban con un tiempo laboral de al menos 3 meses en la Microred Bagua Grande; c) Aquellos que tenían un vínculo contractual vigente. Así mismo se aplicó criterios de exclusión: fueron excluidos aquellos que se encontraban en situación de licencia (con o sin goce de haber), comisión, destaque o vacaciones; aquellos que no quisieron formar parte del estudio ni firmaron consentimiento informado; aquellos que no se encontraban en su puesto laboral al momento de la recopilación de datos.

\subsection{Variables de estudio}

La investigación estuvo compuesta por dos variables:

a) Clima Organizacional (variable independiente). La cual consto de tres dimensiones:

- Ira. Dimensión: Cultura de la organización: Conjunto de valores, creencias y entendimientos importantes que los integrantes de una organización tienen en común. (MINSA, 2012, p.16).

- 2da. Dimensión: diseño organizacional: referida al régimen de actividades o actos conscientemente estructurados de los seres laborales (MINSA, 2012, p.15).

- 3ra. Dimensión: potencial humano: Constituye el sistema social interno de la organización, compuesto por individuos y grupos tanto grandes como pequeños (MINSA, 2012, p.15).

b) Desempeño Laboral (variable dependiente). (Tabla 2).

\subsection{Técnicas e instrumentos}

Técnica de investigación: la técnica investigativa para el estudio del clima laboral, desempeño laboral fue la encuesta; los instrumentos de indagación fueron dos: a) Cuestionario del Clima Organizacional del (MINSA), b) Escala de evaluación del desempeño laboral del (MINSA) segunda versión, la escala de medición fue sometida a mejoras que consistieron en la reducción de número de ítem. Así mismo se realizó una encuesta piloto en el 20\% (13) 
trabajadores para la verificación de su confiabilidad antes de aplicar a toda la población estructural del estudio (60). Para la aplicación de las fichas de evaluación de desempeño laboral se tomaron en cuenta 4 perfiles:

Tabla 2. Dimensiones de la variable desempeño laboral

\begin{tabular}{|c|c|c|c|c|}
\hline Dimensión & Directivos & Profesionales & Técnicos & Auxiliares \\
\hline lra & Planificación & Planificación & Responsabilidad & Responsabilidad \\
\hline $2 \mathrm{da}$ & $\begin{array}{c}\text { Dirección y } \\
\text { organización }\end{array}$ & Responsabilidad & Iniciativa & Iniciativa \\
\hline $3 \mathrm{ra}$ & $\begin{array}{l}\text { Supervisión y } \\
\text { control }\end{array}$ & Iniciativa & Claboración & $\begin{array}{l}\text { Calidad del } \\
\text { Trabajo }\end{array}$ \\
\hline $4 \mathrm{ta}$ & $\begin{array}{c}\text { Cumplimiento } \\
\text { de metas }\end{array}$ & Oportunidad & Oportunidad & Oportunidad \\
\hline 5 ta & $\begin{array}{c}\text { Toma de } \\
\text { decisiones }\end{array}$ & $\begin{array}{c}\text { Calidad del } \\
\text { trabajo }\end{array}$ & $\begin{array}{c}\text { Calidad del } \\
\text { trabajo }\end{array}$ & $\begin{array}{c}\text { Buen manejo de } \\
\text { equipos }\end{array}$ \\
\hline $6 \mathrm{ta}$ & Confiabilidad & $\begin{array}{c}\text { Confiabilidad y } \\
\text { discreción }\end{array}$ & $\begin{array}{c}\text { Confiabilidad y } \\
\text { discreción }\end{array}$ & $\begin{array}{c}\text { Confiabilidad y } \\
\text { discreción }\end{array}$ \\
\hline $7 \mathrm{ma}$ & $\begin{array}{c}\text { Relaciones } \\
\text { interpersonales }\end{array}$ & $\begin{array}{c}\text { Relaciones } \\
\text { interpersonales }\end{array}$ & $\begin{array}{c}\text { Relaciones } \\
\text { interpersonales }\end{array}$ & $\begin{array}{c}\text { Relaciones } \\
\text { interpersonales }\end{array}$ \\
\hline $8 \mathrm{va}$ & $\begin{array}{l}\text { Cumplimiento } \\
\text { de normas }\end{array}$ & $\begin{array}{c}\text { Cumplimiento } \\
\text { de metas }\end{array}$ & $\begin{array}{c}\text { Cumplimiento } \\
\text { de metas }\end{array}$ & $\begin{array}{l}\text { Cumplimiento } \\
\text { de normas }\end{array}$ \\
\hline
\end{tabular}

Adaptado de: MINSA, (2008).

- Directivo (formato Nro. 1): comprendió las evaluaciones de aquellos que por designación de las autoridades con competencia desempeñan cargos de confianza.

- Profesional (formato Nro. 2): comprendió las evaluaciones de quienes tienen título profesional.

- Técnico (formato Nro. 3): comprendió la evaluación de aquellos que cuentan con formación superior y/o universitario de tipo inconcluso o poseedores de capacitación de tipo tecnológica o experiencias técnicas con reconocimiento formal, tienen funciones como técnicos y se posicionan en el Grupo Ocupacional de Técnicos. 
- Auxiliar (formato Nro. 4): comprendió la evaluación de quienes poseen educación de nivel secundario y/o experiencias calificadas para el ejercicio de puestos de apoyo dentro del Grupo Ocupacional Auxiliar.

\subsection{Análisis de datos}

El análisis y procesamiento de la información recogida se llevó a cabo en el software Statistical Package for Social Sciences (SPSS) en español, versión nro. 25, para el análisis descriptivo de variables de índice cualitativas fueron empleadas frecuencias absolutas y relativas (\%). El análisis bivariado se realizó mediante aplicación de la prueba Chi-cuadrado con un nivel de confianza del 95\%, siendo considerado un p-valor menor a 0.05 como "significativo"; asimismo, se empleó el coeficiente de correlación de Spearman para la determinación de la intensidad y direccionalidad de dicha correlación.

\section{Resultados}

Tabla 3. Características laborales de los trabajadores de acuerdo a la edad, tiempo laboral, tipo de contrato y categoría profesional de la Microred de Salud Bagua Grande

\begin{tabular}{|r|c|c|}
\hline & $\mathbf{N}$ & $\%$ \\
\hline $20-29$ años & 15 & 25.0 \\
\hline $30-39$ años & 28 & 46.7 \\
\hline $30-49$ años & 17 & 28.3 \\
\hline $1-4$ año s & 60 & 100.0 \\
\hline $5-9$ año s & 28 & 46.7 \\
\hline $10-14$ años & 21 & 35.0 \\
\hline $15-19$ años & 5 & 6.7 \\
\hline 20 a más & 2 & 8.3 \\
\hline Tiempo laboral & 60 & 3.3 \\
\hline CAS & 31 & 100.0 \\
\hline Nombrado & 25 & 51.7 \\
\hline Funcional 276 & 2 & 41.7 \\
\hline Otros & 2 & 3.3 \\
\hline Tipo de contrato & 60 & 3.3 \\
\hline Categoría profesional & 24 & 40.0 \\
\hline Tec. Enfermería & 9 & 15.0 \\
\hline Obstetra & 8 & 13.3 \\
\hline Enfermera & 7 & 11.7 \\
\hline Médico & & \\
\hline
\end{tabular}


REVISTA DE LA UNIVERSIDAD DEL ZULIA. 3ª época. Año 11 N 31, 2020 Clarita Melissa Puitiza Lucano et al/// Clima organizacional y desempeño laboral ... 71-85

DOI: http://dx.doi.org/10.46925//rdluz.31.06

\begin{tabular}{|r|c|c|}
\hline Tec. Laboratorio & 5 & 8.4 \\
\hline Odontóloga & 2 & 3.3 \\
\hline Tec. Contabilidad & 2 & 3.3 \\
\hline Tec. Comp. e Informat. & 1 & 1.7 \\
\hline Psicóloga & 1 & 1.7 \\
\hline Otro & 1 & 1.7 \\
\hline Total & 60 & 100.0 \\
\hline
\end{tabular}

Tabla 4. La dimensión del clima organizacional, la cultura de la organización fue considerada como regular (66.7\%) por trabajadores de la Microred de Salud Bagua Grande. Solo el 18.3\% consideraron la cultura como adecuada y un 15.0\% lo consideró inadecuado. En la dimensión 2 el diseño organizacional fue considerado como regular (40.0\%), en tercer lugar, solo el 33.3\% lo consideraron como adecuado y un 26.7\% lo consideró inadecuado. En la dimensión 3 del clima organizacional el potencial humano, fue considerado como regular (43.3\%), en segundo lugar, el 38.3\% de trabajadores los consideraron adecuado y de igual manera el 28.3\% lo consideró como inadecuado.

Tabla 4. Dimensiones del clima organizacional según trabajadores de la Microred de Salud Bagua Grande. 2019

\begin{tabular}{|c|c|c|c|c|c|c|}
\hline \multirow{3}{*}{ Dim. 1: Cultura organizacional } & \multicolumn{2}{l|}{$\begin{array}{l}\text { Dim. 2: Diseño } \\
\text { organizacional }\end{array}$} & \multicolumn{2}{c|}{$\begin{array}{c}\text { Dim. 3: Potencial } \\
\text { humano }\end{array}$} \\
\hline \multirow{2}{*}{ Adecuado } & $\mathbf{n}$ & $\%$ & $\mathbf{n}$ & $\%$ & $\mathbf{n}$ & $\%$ \\
\cline { 2 - 7 } & 11 & 18.3 & 20 & 33.3 & 17 & 28.3 \\
\hline Regular & 40 & 66.7 & 24 & 40.0 & 26 & 43.3 \\
\hline Inadecuado & 9 & 15 & 26 & 26.7 & 17 & 28.3 \\
\hline Total & 60 & 100 & 60 & 100 & 60 & 100 \\
\hline
\end{tabular}

Fuente: Elaboración propia

Tabla 5. El clima organizacional fue considerado como regular por el $45.0 \%$ de trabajadores de la Microred de Salud Bagua Grande. Asimismo, el 28.3\% de trabajadores de la microred consideraron al clima como adecuado; mientras que un 26.0\% lo consideró como inadecuado. El desempeño laboral fue considerado como regular por el $71.7 \%$ de trabajadores de la Microred de Salud Bagua Grande. En segundo lugar, solo el 15.0\% de trabajadores consideraron al desempeño laboral como adecuado; mientras que un 13.0\% lo consideró como inadecuado. 
Según la tabla 06, se encontró que existe asociación ( $\mathrm{p}=0.001$ ) entre la cultura de la organización y el desempeño laboral. Asimismo, dicha relación es directa, entendiéndose que cuanto mejor es la cultura de la organización mejor el desempeño laboral de los trabajadores, y viceversa, siendo dicha relación de intensidad moderada (Coef. Pearson: 0.407). Además, los resultados explican que existe asociación $(\mathrm{p}=0.001)$ entre el diseño organizacional con el desempeño laboral.

Tabla 05. Nivel del clima organizacional según trabajadores de la Microred de Salud Bagua Grande. 2019

\begin{tabular}{|c|c|c|c|c|c|}
\hline \multicolumn{2}{|c|}{ Clima organizacional: Global } & \multicolumn{3}{c|}{ Desempeño laboral } \\
\hline \multirow{3}{*}{ Adecuado } & $\mathbf{n}$ & $\%$ & & $\mathbf{n}$ & $\%$ \\
\cline { 2 - 6 } & 17 & 28.3 & Adecuado & 9 & 15 \\
\hline Regular & 27 & 45.0 & Regular & 43 & 71.7 \\
\hline Inadecuado & 16 & 26.7 & Inadecuado & 8 & 13.3 \\
\hline Total & 60 & 100.0 & & 60 & 100.0 \\
\hline
\end{tabular}

Fuente: Elaboración propia

Tabla 06. Correlación entre las dimensiones de la cultura organizacional, diseño organizacional y potencial humano en los trabajadores de la Microred de Bagua Grande. 2019

\begin{tabular}{|c|c|c|c|}
\hline \multicolumn{4}{|c|}{ DESEMPEÑO LABORAL } \\
\hline Dimensión & $\mathrm{n}$ & $\begin{array}{c}\mathrm{p}^{-} \\
\text {valor }\end{array}$ & $\begin{array}{l}\text { Coef. } \\
\text { Pearson }\end{array}$ \\
\hline Dimensión l: Cultura de la organización & 60 & 0.001 & 0.432 \\
\hline Dimensión 2: Diseño organizacional & 60 & 0.001 & 0.425 \\
\hline Dimensión 3: Potencial humano & 60 & 0.060 & 0.244 \\
\hline
\end{tabular}

Fuente: Elaboración propia

\section{Discusión}

Los resultados indican que el clima organizacional, en los trabajadores de la Microred Sanitaria Bagua Grande lo consideraron como regular (46.7\%). Asimismo, un 28.3\% lo consideró inadecuado y solo la cuarta parte dijo que era adecuada (25.0\%). Este resultado coincide con lo reportado por Aleman (2018), quien reportó en un hospital MINSA del interior del país un clima 
organizacional mayoritariamente regular (61.8\%), siendo en $7.3 \%$ y $30.9 \%$ de casos considerado un mal y buen clima laboral, respectivamente. Peña et al. (2015) muestran que el clima organizacional es mayormente percibido como regular (36.8\%); asimismo, un clima organizacional inadecuado ("bajo") se encuentra en poco más de la tercera parte de casos (35.6\%) y el clima adecuado ("alto") es encontrado en poco más de la cuarta parte de casos (27.6\%). Los estudios con los cuales se contrasta el nivel de clima organizacional encontrado en la microred sanitara demuestran mucha similitud al hallar situaciones regulares o promedio, lo cual evidencia que aún queda espacio para la realización de mejoras. Peña et al. (2015), evaluó el clima organizacional con una metodología distinta; esto hace pensar que independientemente del método con que se evalúe al clima, el resultado es similar.

Los resultados evidencian que en la Microred de Salud Bagua Grande se encontró un clima organizacional inadecuado por el $26.7 \%$ de los trabajadores. En lo que respecta a los elementos (dimensiones) que conforman el clima organizacional, se encontró una cultura organizacional (dimensión 1) considerada inadecuada por un $15.0 \%$ de trabajadores, lo cual es indicador de la existencia de desfavorables e inadecuados patrones de comportamiento, creencias y valores en común por los miembros de la Microred de Salud Bagua Grande. Por otro lado, un $18.3 \%$ consideró este elemento como adecuado y el $66.7 \%$ de trabajadores percibieron la cultura organizacional como regular.

En lo que respecta a los otros componentes del clima organizacional, se encontró un diseño organizacional (dimensión 2) percibido como regular por el 40.0\% de trabajadores de la microred e inadecuado por un 26.7\% de estos; hallazgo similar a lo encontrado por Solís (2017). Requejo (2017) en un hospital de Tarapoto encontró que el diseño organizacional fue percibido como regular por el $60.4 \%$ de trabajadores y solo en un $13.4 \%$ de casos fue considerado favorable. Dado que el diseño comprende aspectos remunerativos, toma de decisiones, comunicación y estructura, son estos puntos donde resulta necesario trabajar a nivel de microred. Al respecto de estos indicadores del diseño organizacional, en un estudio realizado en el Hospital Santa Rosa (Lima, Perú) se encontró que las condiciones remunerativas fue el aspecto peor valorado $(72.3 \%)$, seguido de la toma de decisiones (42.8\%), siendo la comunicación organizacional y la estructura los indicadores con valoraciones más favorables (MINSA, 2015). 
Con respecto al potencial humano (dimensión 3), este fue considerado como regular por un 43.3\% de trabajadores de la Microred de Salud Bagua Grande, lo cual es similar a lo encontrado por Requejo (2017) para quien también el potencial humano fue considerado como regular por hasta $53.1 \%$ de los trabajadores sanitarios a quienes encuestó. Asimismo, otros estudios realizados en Lima en el Hospital Santa Rosa reportan que el potencial humano se encontró en condiciones regulares en un 51.0\% de casos (MINSA, 2015).

De manera general se encontró que el clima organizativo si guarda correlación estrecha con el desempeño en el centro de labores del trabajador de la Microred de Salud Bagua Grande ( $\mathrm{p}=0.001$; Rho: 0.414); asimismo, dicha correlación es significativa, directa y de intensidad moderada. Ello quiere decir que cuanto mejor percibido es el clima organizacional por los trabajadores, mejor es su desempeño; mientras que, cuanto peor (o más desfavorable) sea percibido el clima menor será el desempeño laboral.

Este hallazgo es similar a lo encontrado en un Hospital de Chancay en el 2018 y realizado sobre 564 trabajadores, encontrándose que el clima organizativo laboral se vincula de manera significativa y estrecha ( $\mathrm{p}<0.001)$ con el desempeño en el centro laboral (Altamirano et al. 2018). No obstante, este hallazgo investigativo corresponde a un estudio realizado en un solo establecimiento y no en una microred, hecho que podría restar credibilidad a la similitud aparentemente existente entre el presente estudio y la investigación de Altamirano et al. (2018);

Otros investigadores peruanos ejecutaron un estudio científico en la Microred de salud Leonor Savedra de Lima y corroboraron lo encontrado en esta investigación, vale decir vínculo íntimo entre clima organizativo del centro laboral y el desempeño del cuerpo de trabajadores (p<0.05; Rho: 0.414) (Chinga \& Terrazas, 2017). No obstante, dicha relación es más intensa que la encontrada en el presente estudio, al punto de poner al clima organizacional como factor determinante para el desempeño laboral. Aunque sería deseable contrastar con investigaciones realizadas a nivel macro asistencial, tal como hizo Chinga y Terrazas (2017) en una microred de Lima, la mayor parte de estudios revisados -todos en contextos organizacionales sanitarioscorroboran las conclusiones obtenidas, algunos han sido realizados en unidades hospitalarias específicas (Pujay, 2017). En general se podría establecer que el clima organizativo e institucional si influye grandemente sobre el desempeño de la fuerza laboral. 
Con respecto a los tres componentes del clima organizacional, solo resultaron influir en el desempeño la cultura organizacional (dimensión l: p=0.001; Rho: 0.407) y el diseño organizacional (dimensión 2: $\mathrm{p}=0.001$; Rho: 0.425). El primero referido a la identidad (sentimiento de pertenencia a la microred), conflicto/cooperación (colaboración y apoyo entre trabajadores) y motivación; y el segundo referido a la estructura (reglas, procedimientos y burocracia organizacional), toma de decisiones (consideración de quienes conforman la fuerza laboral en las tomas de decisiones), organizativo (redes de comunicación) y remuneración. Son todos estos atributos pormenorizados los que influyen sobre el desempeño del trabajador y en los cuales es necesario trabajar para elevar el desempeño laboral, y consecuentemente propiciar una mejor calidad de servicios, así como incrementar la eficiencia en la prestación en la atención de la población adscrita a la microred.

Por otro lado, el potencial humano (dimensión 3) es un elemento del clima organizacional referido al liderazgo (influencia de jefes), innovación (voluntad de cambio), recompensa (uso del premio más que el castigo) y confort (comodidad del ambiente físico), los cuales según la presente investigación no se relacionó con el desempeño laboral ( $p=0.060 ;$ Rho: 0.244). En este sentido, corresponden a atributos para mejorar el desempeño laboral sí podrían influir sobre otros desenlaces como satisfacción laboral, compromiso organizacional, estrés laboral, calidad de servicios, etc. Así estudios realizados en México indican que alguno de estos atributos podría influir sobre la calidad de servicios (Sotelo \& Figueroa, 2017).

\section{Conclusiones}

El clima organizacional se relaciona de manera estrecha y estadísticamente significativa con el desempeño laboral; asimismo, dicha relación es directa y de intensidad moderada, por lo cual se concluye que cuando hay un buen clima organizativo es mejor el desempeño laboral de los trabajadores de la Microred de Salud Bagua Grande. Asimismo, la cultura de la organización y el diseño organizacional se relacionaron de manera significativa con el desempeño laboral, lo cual no sucedió para el potencial humano estos resultados indicaron que el desempeño laboral fue regular según percepción del $71.7 \%$ de trabajadores de la Microred de Salud Bagua Grande; sin embargo, en un $13.3 \%$ fue considerado como inadecuado y solo en un $15.0 \%$ de casos se le 
REVISTA DE LA UNIVERSIDAD DEL ZULIA. 3ª época. Año 11 N 31, 2020 Clarita Melissa Puitiza Lucano et al/// Clima organizacional y desempeño laboral ... 71-85

DOI: http://dx.doi.org/10.46925//rdluz.31.06

percibió como adecuado. El clima organizacional fue regular según percepción del $46.7 \%$ de trabajadores de la Microred de Salud Bagua Grande; sin embargo, en un 28.3\% fue considerado como inadecuado y solo en un $25.0 \%$ de casos se le percibió como adecuado.

La fuerza laboral conformada por trabajadores de la Microred de Salud Bagua Grande se caracterizó por encontrarse en el grupo etario d e 30 a 39 años (46.7\%), tener un tiempo laboral de 1 a 4 años (46.7\%), en condición laboral CAS (51.7\%), así como pertenecer a la categoría profesional técnico de enfermería (40.0\%). El cual se debe mejorar el desempeño laboral y de esa manera incrementar la prestación de servicios a la comunidad.

\section{Referencias}

Aleman, G. (2018). Clima laboral y evaluación del desempeño en personal de salud, involucrado en actividades administrativas de los hospitales MINSA de Arequipa 2017. (Tesis de Maestría), Universidad Nacional de San Agustín de Arequipa, Arequipa.

Altamirano, M., Galvez, M., \& Inche, F. (2018). Clima organizacional y desempeño laboral de los trabajadores del Hospital de Chancay, Huaral, Lima 2018. Tesis de grado, Universidad Inca Garcilazo de la Vega, Facultad de Ciencias Administrativas, Lima, Perú.

Aliaga, M., \& Julcamoro, R. (2016). Influencia del clima organizacional en el desempeño laboral de los colaboradores del Hospital Regional de Cajamarca en el área de hospitalización, 2016. (Tesis de grado), Universidad Privada del Norte, Cajamarca.

Ballard, M., \& Montgomery, P. (2017). Systematic review of interventions for improving the performance of community health workers in low-income and middle-income countries. Systematic review of interventions for improving the performance of community health workers in low-income and middle-income countries., 7(10), e014216. doi:10.1136/bmjopen-2016-014216.

Chinga, L., \& Terrazas, S. (2017). Clima organizacional y el desempeño laboral de los trabajadores de salud de la Micro Red de Salud "Leonor Saavedra-Villa San Luis" 2014. (Tesis de Maestría en Gestión de Servicios de Salud), Universidad César Vallejo, Lima.

Espinoza, C. (2018). Clima organizacional y desempeño laboral en el hospital de Huaral- 2016. (Tesis de Maestría en Salud Pública), Universidad César Vallejo, Lima.

Gobierno Regional de Amazonas. (2015). Plan Operativo Institucional 2015. Red de Salud Amazonas, Amazonas, Bagua. 
REVISTA DE LA UNIVERSIDAD DEL ZULIA. 3ª época. Año 11 N 31, 2020 Clarita Melissa Puitiza Lucano et al/// Clima organizacional y desempeño laboral ... 71-85

DOI: http://dx.doi.org/10.46925//rdluz.31.06

García, O. (2016). Relación entre el clima organizacional y el desempeño laboral de trabajadores del Hospital Belén de Trujillo - 2016. (Tesis de grado), Universidad Autónoma de Ica, Chincha.

Gonzáles, A. (2006). Métodos de compensación basados en competencias. Bogotá, Colombia: Ediciones Uninorte.

Irimu, G., Greene, A., Gathara, D., Kihara, H., Maina, C., Mbori, D. (2014). Factors influencing performance of health workers in the management of seriously sick children at a Kenyan tertiary hospital - participatory action research. BMC Health Serv Res, 14(9), 59. doi:10.1186/1472-6963$14-59$.

Ministerio de Salud (2016). Hacia la cobertura universal en salud. Conferencia Internacional. MINSA, Viceministerio de Prestaciones y Aseguramiento en Salud, Lima, Perú.

Ministerio de Salud (2008). Normas y procedimientos para el proceso de evaluación de evaluación de desempeño y conducta laboral. MINSA, Oficina General de Recursos Humanos. Lima, Perú: Directiva Administrativa N¹42 .

Ministerio de Salud. (2012). Metodología para el Estudio del Clima Organizacional v.02. MINSA, . Lima, Perú: Dirección General de Salud de las Personas.

MINSA. (2015). Informe de aplicación de encuesta de clima organizacional. Hospital Santa Rosa 2015. Hospital Santa Rosa, Oficina de Gestión de la Calidad, Lima, Perú.

Peña, R., Hernández, D., Vélez, A., García , M., Reyes, M., \& Ureña, E. (2015). Clima organizacional de equipos directivos y su relación con los resultados en la atención a la salud. Salud Pública de México, 57(6), 528-536.

Pujay, A. (2017). Clima laboral y desempeño de los trabajadores del Servicio Centro Quirúrgico del Hospital II Pasco - 2017. (Tesis de segunda especialidad), Universidad Nacional Hermilio Valdizán, Huánuco.

Picoy, D. (2015). Clima organizacional en establecimientos de salud de la Micro Red Villa en el año 2014. Rev Fac Med URP, 15(3), 34-39.

Requejo, C. (2017). Clima organizacional en el personal de salud del Hospital de Contingencia II-2 de Tarapoto, San Martín, abril-setiembre, 2016. (Tesis), Universidad Nacional de San Martín, Facultad de Medicina, Tarapoto, Perú

Solís, Z. (2017). Clima organizacional en los trabajadores del Hospital de Baja Complejidad Vitarte, 2013. (Tesis de Maestría en Gerencia de Servicios de Salud), Universidad Nacional Mayor de San Marcos, Unidad de Posgrado, Lima.

Sotelo, J., \& Figueroa, E. (2017). El clima organizacional y su correlación con la calidad en el servicio en una institución de educación de nivel medio superior. RIDE. Rev. Iberoam. Investig. Desarro., 8(15), 15-23. 
REVISTA DE LA UNIVERSIDAD DEL ZULIA. 3a época. Año 11 N 31, 2020 Clarita Melissa Puitiza Lucano et al/// Clima organizacional y desempeño laboral ... 71-85

DOI: http://dx.doi.org/10.46925//rdluz.31.06

Sharma, R., Webster, P., \& Bhattacharyya, S. (2014). Factors affecting the performance of community health workers in India: a multi-stakeholder perspective. Glob Health Action, 13(7), 25352. doi:10.3402/gha.v7.25352

Tran, K., Nguyen, P., Dang, T., \& Ton, T. (2018). The Impacts of the High-Quality Workplace Relationships on Job Performance: A Perspective on Staff Nurses in Vietnam. Behav Sci (Basel), 8(12), 109. doi:10.3390/bs8120109

Weldegebriel, Y., Ejigu, Y., Weldegebreal, F., \& Woldie, M. (2016). Motivation of health workers and associated factors in public hospitals of West Amhara, Northwest Ethiopia. Patient Prefer Adherence, 10(5), 159-169. doi:10.2147/PPA.S90323. 